\title{
Tratamento de água com hidrocarbonetos aromáticos por uso de reator em bateladas sequenciais com inoculo fúngico
}

\section{Treatment of water with aromatic hydrocarbons by use of sequential batch reactor with fungi inoculum}

\begin{abstract}
Kelly Rodrigues
Engenheira Civil pela Universidade Estadual do Maranhão (UEMA) - São Luís (MA), Brasil. Doutora em Hidráulica e Saneamento pela Escola de Engenharia de São Carlos da Universidade de São Paulo (USP) - São Carlos (SP), Brasil. Professora Efetiva do Departamento da Área de Química e Meio Ambiente e do Programa de Pós-graduação em Gestão e Tecnologia Ambiental do Instituto Federal de Educação, Ciência e Tecnologia do Ceará (IFCE) - Fortaleza (CE), Brasil.
\end{abstract}

\section{Patrícia Celestino Carvalho de Oliveira}

Tecnóloga em Gestão Ambiental pelo IFCE. Mestranda do Programa de Pós-graduação em Gestão e Tecnologia Ambiental do IFCE - Fortaleza (CE), Brasil.

\section{Francisco Wilame Amaral Júnior}

Tecnólogo em Gestão Ambiental pelo IFCE. Mestre em Gestão e Tecnologia Ambiental pelo Programa de Pós-graduação do IFCE - Fortaleza (CE), Brasil.

\section{João Paulo da Silva Siqueira}

Tecnólogo em Saneamento pelo IFCE - Fortaleza (CE), Brasil. Mestrando em Recursos Hídricos e Saneamento pela Universidade Federal de Alagoas (UFAL) Maceió (AL), Brasil.

\section{Rinaldo dos Santos Araújo}

Químico Industrial e Doutor em Química Orgânica pela Universidade Federal do Ceará (UFC) - Fortaleza (CE), Brasil. Professor Efetivo do Departamento da Área de Química e Meio Ambiente e do Programa de Pós-graduação em Gestão e Tecnologia Ambiental do IFCE - Fortaleza (CE), Brasil.

\section{Carlos Ronald Pessoa Wanderley}

Engenheiro Civil e mestre em Engenharia Hidráulica e Ambiental pela UFC - Fortaleza (CE), Brasil. Professor Efetivo do Departamento da Área de Química e Meio Ambiente do Campus Maracanaú, IFCE - Maracanaú (CE), Brasil.

\section{Glória Marinho}

Farmacêutica pela UFC- Fortaleza (CE), Brasil. Doutora em Hidráulica e Saneamento pela Escola de Engenharia de São Carlos da USP - São Carlos (SP), Brasil. Professora Efetiva do Departamento da Área de Química e Meio Ambiente e do Programa de Pós-graduação em Gestão e Tecnologia Ambiental do IFCE - Fortaleza (CE), Brasil.

\section{Resumo}

\begin{abstract}
Um reator em batelada, aerado, com biomassa imobilizada de Aspergillus niger AN400 foi operado durante 10 ciclos de 7 dias para remover benzeno (200 mg. $\left.\mathrm{L}^{-1}\right)$, tolueno (200 mg. $\left.\mathrm{L}^{-1}\right)$ e xileno $\left(50 \mathrm{mg} \cdot \mathrm{L}^{-1}\right)$ - BTX - e de nutrientes de meio basal. O reator era alimentado semanalmente com $4 \mathrm{~L}$ do meio e glicose -1 g. $L^{-1}$, na Fase I, e 0,5 g.L-1 , na Fase II. Os BTX foram detectados até o quarto dia de operação, em todos os ciclos. As melhores eficiências médias de remoção foram na Fase I: 75\%de matéria orgânica solúvel, $80 \%$ de ortofosfato e $77 \%$ de amônia. O reator pode ser uma alternativa viável para tratamento de águas poluídas com BTX, porém há a necessidade de estudar o comportamento do reator durante período de operação mais longo e com ciclos reacionais mais curtos, bem como da identificação dos metabólitos produzidos.
\end{abstract}

Palavras-chave: Aspergillus niger AN 400; bateladas sequenciais; BTX; nutrientes. 


\section{Abstract}

A batch reactor with air and immobilized biomass of Aspergillus niger AN400 was operated during 10 cycles of 7 days to remove benzene (200 mg. $\left.\mathrm{L}^{-1}\right)$, toluene (200 mg. $\mathrm{L}^{-1}$ ), and xylene (50 mg. $\left.\mathrm{L}^{-1}\right)$ - BTX - and nutrients from basal medium. The reactor was weekly fed with $4 \mathrm{~L}$ of the medium and glucose $-1 \mathrm{~g} \cdot \mathrm{L}^{-1}$ (Phase I) and $0.5 \mathrm{~g} . \mathrm{L}^{-1}$ (Phase II). The BTX had been detected until the fourth day of operation in all cycles. The best efficiencies of removal had been in Phase I: $75 \%$ of soluble organic matter, $80 \%$ of orthophosphate and $77 \%$ of ammonia. The reactor can be a viable alternative for the contaminated water treatment with BTX. However it has the necessity to study behavior of the reactor during longer period of operation and with shorter reaction cycles, as well as the identification of the produced metabolites.

Keywords: Aspergillus niger AN 400; sequential batch; BTX; nutrients.

\section{Introdução}

No Brasil, as preocupações relacionadas à poluição de águas subterrâneas por derramamentos de combustíveis vêm crescendo em diversas cidades, como São Paulo, Curitiba, Vitória e Porto Velho (FORTE et al., 2007).

Segundo a Agência Nacional de Petróleo - ANP - (BRASIL, 2009), existem 36.730 postos de combustíveis no país e, muitos destes, operam em condições de risco que podem resultar em grande impacto para o meio ambiente e ao homem, quando da ocorrência de derramamento de combustíveis que apresentam em sua constituição hidrocarbonetos aromáticos, como os compostos BTX, designação para benzeno, tolueno, e xileno (TIBURTIUS et al., 2005).

A grande preocupação em relação aos compostos BTX é em virtude de sua ação impactante no ambiente e dos riscos para a saúde pública, pois o benzeno é carcinogênico, enquanto tolueno e xileno possuem natureza tóxica (FORTE et al., 2007).

O uso de operações físicas e de processos químicos para a remoção dos BTX de águas, além de não apresentar boa eficiência, possui custo mais elevados, gerando ainda subprodutos indesejáveis (FARHADIAN et al., 2008).

Os processos biológicos são os mais utilizados visando à remoção de BTX, porém a presença do etanol pode retardar a biodegradação destes compostos, devido ao consumo de aceptores de elétrons disponíveis no meio (KULKAMP, 2003; TIBURTIUS et al., 2005), sendo que a gasolina brasileira é uma das que apresenta maior percentual de etanol adicionado como cossolvente, de 20 a 26\% (FINOTTI et al., 2009).

Desta forma, visando à otimização de processos existentes e o estudo de novas tecnologias é de fundamental importância em busca de maior eficiência na remoção de BTX. Poucos estudos têm sido realizados sobre a aplicação de fungos em reatores biológicos para remoção de BTX de águas, apesar de que, recentemente, pesquisas têm indicado a habilidade de muitas espécies fúngicas na degradação de hidrocarbonetos aromáticos. Estes relatos têm incentivado o incremento de estudos com o emprego de fungos para a remoção de compostos BTX de águas (KENNES; VEIGA, 2004).

O mecanismo de degradação de poluentes complexos envolve a ação de enzimas como celulases e ligninases, as quais agem sobre compostos recalcitrantes, incluindo os de estrutura aromática, tornando-os acessíveis para a assimilação (RODRIGUES, 2006; SANTOS et al., 2008).

Os fungos dos gêneros Phanerochaete, Paecilomyces, Cladosporium e Pleurotus têm sido empregados em reatores visando à degradação de hidrocarbonetos aromáticos (KENNES; VEIGA, 2004), porém poucos estudos têm sido reportados com a utilização de Aspergillus niger, ainda que várias linhagens desta espécie tenham sido isoladas de áreas contaminadas com petróleo e derivados, o que reflete a necessidade de estudos sobre o conhecimento e a otimização da aplicação desses micro-organismos em reatores biológicos.

$\mathrm{Na}$ busca por novas tecnologias de biorremediação, nesta pesquisa foi utilizada a linhagem Aspergillus niger AN400 em reator em bateladas sequenciais, com o objetivo de remover compostos BTX de água, sob a influência da glicose como cossubstrato, a qual foi adicionada ao meio nas concentrações de 1 g.L.-1 (Fase I) e 0,5 g.L-1 (Fase II). Além disso, também foi avaliada a remoção de macronutientes ( $\mathrm{N}$ e P), os quais foram adicionados ao meio para desenvolvimento dos micro-organismos.

\section{Material e métodos}

\section{Inóculo}

Foi utilizada a linhagem Aspergillus niger AN400, na forma de suspensão de esporos $\left(2 \times 10^{6}\right.$ esporos. $\left.\mathrm{mL}^{-1}\right)$, tendo sido a mesma cultivada em placas de Petri com meio de cultura ágar Sabouraud Dextrose, acrescido de $1 \mathrm{~mL}$ de solução de Vishniac por litro de meio de cultura, a qual foi preparada com água destilada e apresentava a seguinte composição (mg. $\left.\mathrm{L}^{-1}\right): \mathrm{H}_{3} \mathrm{BO}_{3}(50), \mathrm{FeCl}_{2} \cdot 4 \mathrm{H}_{2} \mathrm{O}$ (2.000), $\mathrm{ZnCl}_{2}$ (50), $\mathrm{MnCl}_{2} \cdot 4 \mathrm{H}_{2} \mathrm{O}$ (50), $\mathrm{CuCl}_{2} \cdot 2 \mathrm{H}_{2} \mathrm{O}$ (38), $\mathrm{AlCl}_{3}$. $\mathrm{H}_{2} \mathrm{O}(90), \mathrm{CoCl}_{2} \cdot 6 \mathrm{H}_{2} \mathrm{O}$ (2.000). Foi ainda acrescido ao meio 0,05 g. $\mathrm{L}^{-1}$ de cloranfenicol, a fim de minimizar o crescimento de bactérias.

As placas permaneceram à temperatura de $\pm 28^{\circ} \mathrm{C}$, durante 10 dias, e, após esse período, os esporos foram removidos com auxílio de alça estéril e $4 \mathrm{~mL}$ de solução de Tween 80. Posteriormente, a suspensão de esporos foi transferida para tubos de ensaio.

Para contagem dos esporos, foi preparada solução com $50 \mu \mathrm{L}$ da suspensão previamente agitada em agitador tipo vortex, acrescidos de $950 \mu \mathrm{L}$ de solução Tween 80, resultando em diluição de 1:20. Em 
seguida, foram transferidos $20 \mu \mathrm{L}$ da solução preparada para câmara de Neubauer para realizar a contagem dos esporos em microscópio óptico com aumento de 40 vezes.

\section{Composição do meio}

O meio foi preparado com água de torneira, previamente esterilizada, em autoclave por 15 minutos, adicionada de solução de nutrientes (g. $\left.\mathrm{L}^{-1}\right)$ : $\left(\mathrm{NH}_{4}\right)_{2} \mathrm{SO}_{4}(0,5) ; \mathrm{NaNO}_{3}(0,25) ; \mathrm{KH}_{2} \mathrm{PO}_{4}(0,20)$; $\mathrm{MgSO}_{4}(0,25) ; \mathrm{CaCl}_{2} \cdot 2 \mathrm{H}_{2} \mathrm{O}(0,01) ; \mathrm{CuSO}_{4} .7 \mathrm{H}_{2} \mathrm{O}(0,08) ; \mathrm{H}_{2} \mathrm{MoO}_{4}$ $(0,05) ; \mathrm{MnSO}_{4} .5 \mathrm{H}_{2} \mathrm{O}(0,05) ; \mathrm{Fe}_{2}(\mathrm{SO} 4)_{3}(0,05) ; \mathrm{ZnSO}_{4}(0,04)$.

As concentrações dos compostos BTX propostas foram: $200 \mathrm{mg} . \mathrm{L}^{-1}$ de benzeno, $200 \mathrm{mg} . \mathrm{L}^{-1}$ de tolueno e $50 \mathrm{mg} . \mathrm{L}^{-1}$ de xileno. Foram adicionados também $2000 \mathrm{mg} \cdot \mathrm{L}^{-1}$ de etanol, visando à melhor solubilização dos compostos, bem como de 0,1 g.L-1 ${ }^{-1}$ de cloranfenicol, para minimizar a ação de bactérias.

\section{Imobilização da biomassa fúngica}

A biomassa foi imobilizada em manta de polipropileno $(2 \times 2 \mathrm{~cm})$ acondicionados em redes de polietileno no interior de frascos erlenmeyer de $250 \mathrm{~mL}$. As redes contendo a mantasuporte foram imersas em $200 \mathrm{~mL}$ de meio de crescimento, adicionando-se 5 g.L $\mathrm{L}^{-1}$ de glicose - fonte de carbono e energia - e cloranfenicol $\left(0,10\right.$ g. $\left.\mathrm{L}^{-1}\right)$. Os frascos permaneceram sob agitação de $150 \mathrm{rpm}$, durante 72 horas, de modo que, ao completar 24 horas, o meio antigo foi substituído por um recém preparado. Após a imobilização da biomassa, os fungos imobilizados na manta suporte foram transferidos para o reator em bateladas sequenciais para partida do mesmo.

\section{Montagem e operação do reator em bateladas sequenciais}

O reator utilizado era de vidro e possuía volume total de $5 \mathrm{~L}$, tendo sido alimentado com 4 L do meio aquoso com BTX. A aeração foi mantida por uso de minicompressores. O reator foi coberto com sacos pretos de polietileno durante todo o experimento a fim de evitar possíveis efeitos da ação da luz ambiente sobre a fotodegradação dos poluentes.

O sistema havia sido anteriormente operado com meio contendo os compostos BTX em concentração de 5 g. $\mathrm{L}^{-1}$ de glicose (dados não apresentados), por um período total de 45 dias, após o qual o reator passou a ser alimentado com concentrações menores de glicose, de 1 g.L.- (Fase I) e 0,5 g.L L $^{-1}$ (Fase II). A operação do reator nas Fases I e II abrangeu período de 70 dias, nos quais o reator foi operado em ciclos de 7 dias, sendo 4 ciclos na Fase II e 6 ciclos na Fase II.

No início de cada ciclo, o reator era alimentado com meio aquoso recém-preparado e eram retiradas alíquotas diárias para monitoramento e efetuadas análises de: demanda química de oxigênio (DQO), benzeno, tolueno, xileno, $\mathrm{pH}$, amônia, nitrito, nitrato e ortofosfato.
As análises foram executadas segundo APHA (1995), exceto nitrato — realizada de acordo com Rodier (1975) - e benzeno, tolueno e xileno, cuja determinação ocorreu por cromatografia líquida (HPLC), empregando coluna C8, fase móvel constituída de mistura de $80 \%$ metanol/20\% água, modo isocrático e comprimento de onda de 200 $\mathrm{nm}$ para benzeno e $207 \mathrm{~nm}$ para tolueno e xileno.

\section{Análise de microscopia}

Ao final da operação do reator, foram retiradas amostras da espuma de poliuretano contendo biomassa aderida que foi então removida por atrito com pérolas de vidro em erlenmeyer contendo água destilada, o qual foi agitado em vortex a fim obter o desprendimento do biofilme.

Em seguida, fizeram-se diluições de 1:10, 1:100 e 1:1000, com água destilada e esterilizada a $120^{\circ} \mathrm{C}$, durante 15 minutos, e adicionou-se $\mathrm{NaCl}(0,9 \% \mathrm{v} / \mathrm{v})$, tendo-se transferido $1 \mathrm{~mL}$ desta diluição para placas de Petri, contendo meio de cultura Saboraund Dextrose. As placas foram mantidas à temperatura $\pm 27^{\circ} \mathrm{C}$, durante período de 7 dias para verificação das colônias. Amostras dos fungos em placas foram fixadas em lamínulas e submetidas à observação em microscópio óptico ACROM 1000, aumento de 1.600 vezes, com câmera de captura de imagem para identificação morfológica dos micro-organismos.

\section{Ensaios abióticos}

\section{Ensaio de volatilização}

Foi utilizado frasco de vidro com volume total de $3 \mathrm{~L}$, vedado com tampa apropriada e coberto com sacos pretos de polietileno. O frasco foi preenchido com 2 L de água de destilada acrescida de benzeno e tolueno, na concentração de $200 \mathrm{mg} \cdot \mathrm{L}^{-1}$, e xileno, na concentração de $50 \mathrm{mg} \cdot \mathrm{L}^{-1}$. O etanol foi adicionado ao meio na concentração de $2.000 \mathrm{mg} \cdot \mathrm{L}^{-1}$.

O conteúdo líquido no interior do frasco foi mantido agitado com uso de minicompressor de ar. Foram retiras alíquotas para determinação da concentração de benzeno, tolueno e xileno no meio líquido ao longo de 7 dias. Diariamente foram realizadas coletas de amostras nos seguintes horários: 9h30, 11h30, 15h30 e 17h30.

Ensaio de adsorção

Adicionou-se a um frasco de vidro de $2 \mathrm{~L}, 21 \mathrm{~g}$ da manta suporte, na forma de quadrados de 2 x $2 \mathrm{~cm}$, conforme efetuado na montagem do reator, porém sem imobilização dos micro-organismos. O frasco recebeu 1 L de água de destilada acrescida de benzeno, tolueno, xileno e etanol nas mesmas concentrações utilizadas no ensaio de volatilização.

O monitoramento da concentração dos BTX foi realizado ao longo de 10 horas, sendo retiradas alíquotas a cada 1 hora a fim de verificar a concentração de saturação e a partir desta, estimada a massa 
total de benzeno, tolueno e xileno removida neste ensaio a partir da Equação 1.

$$
\text { Massa do poluente }=(\mathrm{Co}-\mathrm{Ce}) \times \mathrm{V}
$$

Onde,

Co é a concentração inicial;

Ce é a concentração em tempo te $\mathrm{V}$ é o volume útil.

A determinação da concentração de BTX nos ensaios abióticos foi efetuada por meio de cromatografia gasosa usando cromatógrafo gasoso - (GC Perkin Elmer Clarus 500) com detector de ionização de chama (FID), coluna capilar BPX-5 $30 \mathrm{~m}$ x 0,25 mm x 0,25 um, sob as seguintes condições: hélio como gás de arraste $\left(1 \mathrm{~mL} \cdot \mathrm{min}^{-1}\right)$, temperatura do injetor de $280^{\circ} \mathrm{C}$, temperatura do detector de $280^{\circ} \mathrm{C}$, temperatura inicial do forno de $30^{\circ} \mathrm{C}$ e temperatura final de $290^{\circ} \mathrm{C}$. Durante este ensaio o pH do meio também foi monitorado.

\section{Resultados e discussão}

Em ambas as fases estudadas, em todos os ciclos, benzeno e tolueno foram removidos até o quarto dia de operação, sendo que, na Fase II, a detecção destes ocorreu até o segundo dia de tempo reacional. Nas Figuras 1 e 2 são mostradas as variações das concentrações de benzeno, tolueno e xileno ao longo dos ciclos operacionais das Fases I e II, respectivamente.

Segundo Maliyekkal et al. (2004), o tolueno é o primeiro a ser degradado devido ao ataque ao grupo metil por mono-oxigenases. No caso do benzeno, sua desestabilização é mais complexa em relação ao tolueno, pois o anel sozinho é muito estável e, para sua clivagem, é necessário maior reatividade dessa molécula. O grau de dificuldade de degradação aumenta para o xileno, em relação ao tolueno e ao benzeno, devido aos seus isômeros.

Ainda de acordo com aqueles autores, os micro-organismos oxidam primeiro um grupo metil, obtendo-se a formação de intermediários de estrutura molecular ainda complexa. Entretanto, neste trabalho, o xileno foi mais rapidamente removido, até o segundo dia, em ambas as fases, provavelmente, porque a concentração em que se encontrava disponível no meio aquoso era muito baixa, sendo facilmente removido do meio

Em contrapartida, os valores de DQO solúvel não diminuíram ao mesmo tempo em que os de benzeno, tolueno e xileno, como possível resultado da intensa produção de subprodutos oriundos da biotransformação dos compostos BTX.

Na Fase I, até o final de cada ciclo, houve consumo de grande parte desses subprodutos, resultando em remoção média final de matéria orgânica de 75\%. Porém, na Fase II, com a diminuição da concentração da glicose adicionada para 0,5 g.L.-1 , observou-se diminuição da eficiência média (44\%) e acúmulo de matéria orgânica, nos ciclos 9 e 10, sendo importante mencionar que, nestes dois últimos ciclos, a média das concentrações de benzeno e tolueno, no afluente, foram maiores, respectivamente, de $95 \mathrm{mg} \cdot \mathrm{L}^{-1}$ e $71,5 \mathrm{mg} . \mathrm{L}^{-1}$.

Singh (2006) relatou que a adição de cossubstrato facilita o processo de biodegradação, pois sua utilização gera a formação de produtos altamente reativos que se envolvem com compostos menos reativos, como os BTX e alguns intermediários da ruptura de suas moléculas. Assim, a diminuição da adição de glicose (Fase II) pode

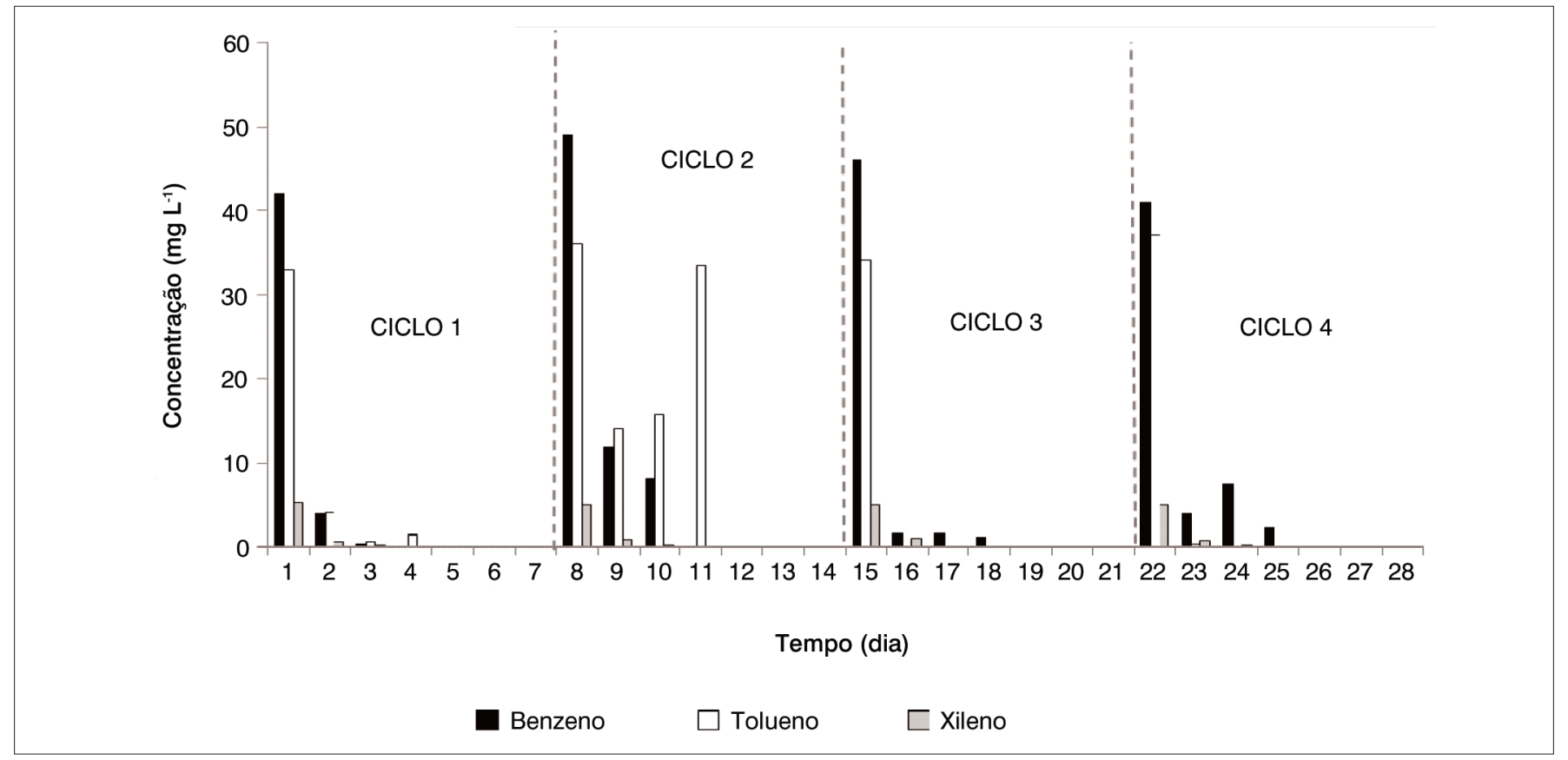

Figura 1 - Variação da concentração de benzeno, tolueno e xileno ao longo dos ciclos operacionais do reator em bateladas sequenciais com inoculo fúngico, na Fase I de alimentação (1 g.L L $^{-1}$ de glicose). 


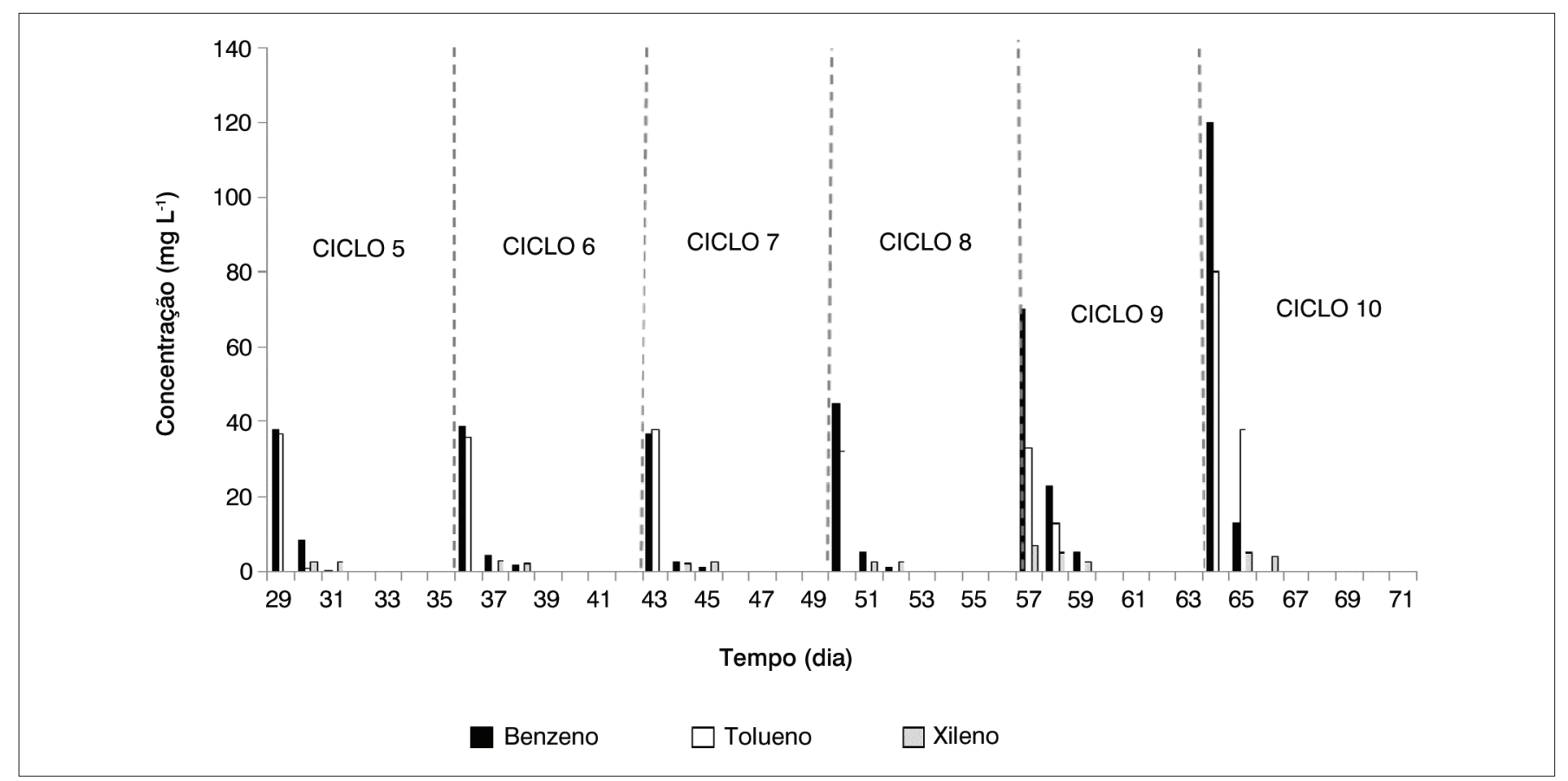

Figura 2 - Variação da concentração de benzeno, tolueno e xileno ao longo dos ciclos operacionais do reator em bateladas sequenciais com inoculo fúngico, na Fase II de alimentação (0,5 g.L $\mathrm{L}^{-1}$ de glicose).

ter contribuído para uma maior dificuldade de assimilação de intermediários da degradação dos BTX pelos fungos.

A análise por cromatografia mostrou que, para o afluente, os picos iniciais de benzeno e tolueno, ocorreram, respectivamente, em 6,12 e 9,4 minutos, sendo que, após 2 e 3 dias, observou-se, além da exaustão de benzeno e tolueno, o surgimento de pico de possível subproduto, em 3,8 minutos, o qual não foi identificado, sendo importante ressaltar que o mesmo poderia estar relacionado tanto coma decomposição dos compostos BTX, como à metabolização pelos micro-organismos dos nutrientes que compuseram o meio.

Entretanto, sabe-se que os fungos filamentosos como Aspergillus niger são capazes de degradar compostos monoaromáticos através do sistema enzimático citocromo P-450 mono-oxigenase, no qual um átomo da molécula de oxigênio presente no meio é incorporado ao substrato aromático, enquanto o outro átomo de oxigênio é reduzido à água. $\mathrm{O}$ óxido de areno formado pode ser metabolizado por duas vias, sendo que em uma delas há a isomerização para formar fenol que pode ser conjugado com sulfato, acido glucônico ou glicose ou ser também metabolizado pelo fungo, conduzindo, principalmente, à formação de catecol e de ácidos orgânicos (REINEKE, 2001).

Em outra via de degradação, o óxido de areno formará dihidrodiol com a configuração trans que pode ser convertido a catecol, metabólito chave da metabolização de vários compostos aromáticos, pois a partir dele ocorre a quebra do anel aromático para futura assimilação. Todas essas reações indicam a grande quantidade de intermediários da biodegradação dos compostos monoaromáticos que podem estar presentes no meio, acumulando-se ou serem novamente degradados a novas moléculas até a formação de $\mathrm{CO}_{2}$ e $\mathrm{H}_{2} \mathrm{O}$, ou seja, a completa mineralização (REINEKE, 2001).

Farias et al. (2008), visando à remoção de $100 \mathrm{mg} . \mathrm{L}^{-1}$ de benzeno de água residuária sintética, contendo 1 g. $\mathrm{L}^{-1}$ de glicose e nutrientes, em reatores em batelada agitada por ação de biomassa dispersa de Aspergillus niger, alcançaram 82,5\% de remoção de DQO em tempo reacional de 20 dias.

$\mathrm{Na}$ presente pesquisa, nos ciclos 3 e 4 (Fase I), ocorreram as maiores remoções de matéria orgânica, de 93 e 88\%, respectivamente, sendo que as mesmas foram alcançadas em tempo reacional muito menor ao utilizado por Farias et al. (2008), de apenas 7 dias, o que foi atribuído à imobilização da biomassa fúngica no meio suporte, o que contribui positivamente na adaptação dos micro-organismos aos compostos tóxicos (PIETRO et al., 2002).

Ao longo da operação do reator, as massas de benzeno, tolueno e de xileno disponibilizadas inicialmente no meio foram, respectivamente, de 2.108, 1.704 e $168 \mathrm{mg}$, tendo-se registrado diferença, entre estes valores e as massas que teoricamente deveriam estar presentes no meio, de 34\% para o benzeno e de $47 \%$ para tolueno e $79 \%$ para o xileno, reforçando ainda mais a perda inicial, particularmente, destes dois últimos.

No caso particular do xileno, este composto apresenta solubilidade baixa em água, de apenas $135 \mathrm{mg} . \mathrm{L}^{-1}$, praticamente insolúvel (MAZZEO et al., 2010). Já o tolueno possui maior dificuldade de dissolução no meio em relação ao benzeno pelo fato do benzeno ser três vezes mais solúvel em água que o tolueno, apresentando solubilidade, respectivamente, de 780 e 535 mg. $\mathrm{L}^{-1}$. 
A presença do etanol na água, o que faz com que aumente a solubilidade dos compostos da gasolina, como benzeno, tolueno e xileno, para a fase aquosa (DONAIRE, 2007).

Assim, a diferença entre a massa dos poluentes que foi efetivamente disponibilizada e a que teoricamente deveria estar no meio indicaria que o etanol, na concentração empregada, não promoveu a solubilização completa dos compostos BTX no meio basal, porém os mesmos, ainda que em concentrações menores, estavam disponíveis no meio para assimilação pelos micro-organismos.

Cabe ressaltar ainda que a perda em relação às concentrações teóricas dos BTX, inicialmente presentes no meio, poderia ser atribuída não apenas pela má dissolução dos compostos estudados, mas também por volatilização.

$\mathrm{O}$ ensaio de volatilização realizado mostrou que o valor do $\mathrm{pH}$ permaneceu em torno de 4,0 $\pm 0,2$ e, até o quarto dia, não houve diminuição da concentração inicial de benzeno, tolueno e xileno, cujas concentrações se mantiveram em $183 \pm 14,175 \pm 6$ e $28 \pm 3$ mg. $\mathrm{L}^{-1}$, respectivamente. Nos últimos dois dias ocorreu a diminuição percentual das concentrações mencionadas, de 15 (benzeno e tolueno) e 22\% (xileno), as quais podem têm sido por volatilização. Contudo, ressalta-se que, durante a operação do reator com fungos, as remoções de BTX ocorreram quase sempre até o terceiro dia, com as maiores remoções, particularmente do primeiro para o segundo dia, não sendo encontrados no meio nos tempos reacionais de cinco, seis e sete dias, o que poderiam endossar a ação biológica como a principal via para a remoção dos compostos BTX do meio.

Além disso, a manta empregada como suporte tinha a capacidade de adsorver 0,024 $\mathrm{g}$ de benzeno e 0,03 $\mathrm{g}$ de tolueno por grama de manta e, como as massas de benzeno e tolueno, removidas ao longo da operação do reator foram, respectivamente, de 0,10 e 0,081 g, verificou-se que, em relação ao benzeno, a remoção via ação biológica foi cerca de 4 vezes superior à sua adsorção pela manta, sendo que, para o tolueno, este valor foi o triplo da capacidade de adsorção do suporte.

É importante ressaltar que o sistema já se encontrava em operação para estudo de fase anterior de alimentação (dados não apresentados) que abrangeu 45 dias de duração, de modo que a massa total de poluentes removidos ao longo da operação do reator pode ter sido ainda maior.

Para o reator com fungos, em relação ao $\mathrm{pH}$, nas fases estudadas ocorreu a diminuição do seu valor de 4,0 até 2,5, ao longo dos ciclos de operação, o que foi atribuído à degradação de benzeno, tolueno e xileno, cuja rota de degradação pelos micro-organismos resulta na formação de ácidos orgânicos, bem como da glicose, cossubstrato adicionado.

Já no primeiro dia de operação, quando foi registrado pH de 2,9, verificou-se remoção elevada dos BTX, tendo o meio permanecido ácido até o último dia de operação, possivelmente, indicando que os ácidos orgânicos produzidos ainda não teriam sido consumidos (KYRIACOU et al., 2005; RODRIGUES et al., 2007).

Jernec e Legisa (2004) afirmaram que em condições de acúmulo de ácidos, como resultado da degradação do substrato, sais de amônio são a fonte nitrogenada preferível pelos fungos e podem ser rapidamente assimilados do meio. Segundo os autores, a espécie Aspergillus niger pode assimilar nitrogênio amoniacal do meio, em culturas em batelada, nas primeiras 24 a 36 horas, em pH 2,5.

Nessa pesquisa foram observadas remoções elevadas de nitrogênio amoniacal, particularmente, na Fase I, quando a concentração inicial de amônia no meio era de 189 mg. L $^{-1}$, alcançando-se percentual médio de remoção de $77 \%$.

Em contraste, o sistema não apresentou bons resultados na remoção de nitrato, inicialmente presente no meio na concentração de 2,9 mg. $\mathrm{L}^{-1}$. Observou-se tendência ao seu acúmulo ao longo de todos os ciclos operacionais.

A remoção do nitrato realizada pelos fungos ocorre em ambiente aeróbio e, diferentemente das bactérias que requerem ambiente anóxico para conversão do nitrato até nitrogênio gasoso, os fungos transformam nitrato em nitrito e este em amônio pela ação das enzimas nitrato e nitrito reductase, sendo este processo denominado de redução desassimilatória do nitrato (GRIFFIN, 1994; ESPOSITO; AZEVEDO, 2001).

Assim, o aumento da concentração de nitrato observado nesse trabalho pode estar relacionado à inibição do processo de redução desassimilatória do nitrato que é realizado pelos fungos sob condições aeróbias. Os fatores que inibem o consumo do nitrato dependem da espécie fúngica, das condições de cultivo e aeração, bem como pela presença de outras fontes de nitrogênio, uma vez que há utilização preferencial de $\mathrm{NH}_{4}^{+}$(ESPÓSITO; AZEVEDO, 2001).

Provavelmente, a concentração inicial de amônia, de 189 mg.L.-1, reprimiu a utilização do nitrato pelos fungos, pois trata-se de concentração alta e supre as exigências nutricionais destes micro-organismos em relação ao nitrogênio, o que não permite a indução do nitrato (GRIFFIN, 1994).

Os bons percentuais de remoção de amônia, registrados na Fase I, podem também ser justificados pela maior disponibilidade da glicose (1 g. $\left.\mathrm{L}^{-1}\right)$. De acordo com Papagianni e Mattey (2004), a glicose é fermentada no ciclo tricarboxílico e forma o composto intermediário $\alpha$-acetogluturato, importante na síntese dos aminoácidos, o qual reage com o íon amônio, retirado do meio e armazenado no interior da célula fúngica, produzindo glutamato, principal aminoácido do metabolismo fúngico, conforme mostrado na Equação 2.

$\mathrm{NH}_{4}^{+}+\alpha$-acetoglutarato $+\mathrm{NADPH} \rightleftarrows$ glutamato + NADP

Desta forma, na Fase I, a maior disponibilidade de glicose resultaria em maior produção de $\alpha$-acetogluturato, favorecendo a síntese de aminoácidos e por consequência a maior utilização do nitrogênio na forma de amônio.

A utilização de $\mathrm{NH}_{4}^{+}$pelos micro-organismos também teria contribuído para a diminuição do valor do $\mathrm{pH}$ do meio, pois, para que este íon atravesse a membrana celular externa e entre na célula fúngica, ocorre simultaneamente a liberação $\mathrm{H}^{+}$do interior da célula para 
o meio extracelular, mediada pela ação da enzima $\mathrm{H}^{+}$-ATPase em sistema de transporte uniport (JERNEC; LEGISA, 2004).

Na Fase II, houve diminuição da eficiência média de remoção de amônia (58\%), o que pode estar relacionado à diminuição da remoção de matéria orgânica carbonácea, registrando-se no final dos ciclos remoção média de 65\%, contra 78\% alcançado na Fase I.

Semelhantemente, na Fase I, também ocorreu remoção mais efetiva de fósforo, em termos de ortofosfato, obtendo-se remoção média de $80 \%$, no final dos ciclos operacionais, sendo que na Fase II, este valor foi de 56\%. Embora muitos autores tenham evidenciado ciclos de consumo e liberação de ortofosfato por fungos (LOPES et al., 2008; MELO et al., 2009), nesse trabalho, seu acúmulo no meio foi observado apenas no ciclo 2 (Fase I). De acordo com Jennings (1995), o ortofosfato é transportado para os vacúolos, onde há formação de polifosfatos, sendo este o principal mecanismo pelo qual os fungos controlam a concentração de fósforo no meio.

$\mathrm{O}$ pH tem grande influência no transporte de fosfato pelos fungos, de modo que valores abaixo de 5,5 podem favorecer o consumo de ortofosfato. Assim, o pH baixo do meio (4 a 2,5) teria contribuído para o alcance das boas remoções de ortofosfato, principalmente na Fase I, quando a remoção de nitrogênio amoniacal foi mais eficiente, uma vez que o metabolismo de assimilação de fósforo está relacionado ao de nitrogênio.

As análises de microscopia comprovaram a presença da espécie Aspergillus niger. No entanto, foi observada a contaminação do reator por outra espécie de fungo, posteriormente identificada como Penicillium sp. Várias espécies do gênero Penicilium, assim como de Aspergillus, têm sido isoladas e identificadas de meios contendo compostos aromáticos como os BTX. Silva et al. (2007) isolaram quatro espécies de fungos filamentosos em água residuária de postos de combustíveis, as espécies isoladas foram: Aspergullus flavus, Cladosporium sp, Penicilium sp e Phoma sp.
Em outra pesquisa, Lemos e Araújo (2002) isolaram e identificaram fungos de água residuária da indústria petrolífera, tendo encontrado fungos dos gêneros Aspergillus, Penicillium, Paecilomyces e Fusarium. Estes estudos endossam a capacidade dos fungos dos gêneros Aspergillus e Penicilium de sobreviverem em meios contendo BTX.

Deste modo, as espécies Aspegillus Níger e Penicillium sp formaram consórcio microbiano que aparentemente foi benéfico para o processo em face da alta remoção de matéria orgânica e de BTX alcançadas, particularmente na Fase I, quando houve maior disponibilidade de glicose.

\section{Conclusões}

O reator em batelada aerado, com inoculo fúngico imobilizado, pode ser uma alternativa para o tratamento de águas contendo BTX, pois estes compostos foram completamente eliminados do meio, quase sempre até o terceiro dia em todos os ciclos operacionais estudados.

A remoção média de nitrogênio amoniacal foi de 77\% (Fase I) e de 44\% (Fase II), resultando em acúmulo de nitrato no meio. A remoção de ortofosfato na Fase I foi de 80\%, diminuindo quando a glicose passou a ser adicionada em menor concentração $\left(0,5\right.$ g. $\left.\mathrm{L}^{-1}\right)$, contudo não foi observado seu acúmulo no meio - no interior do reator - exceto na fase anterior, no ciclo 2 .

As médias dos percentuais alcançados no final dos ciclos das Fases I e II, em relação à matéria orgânica dissolvida, foram, respectivamente, de 74 e 44\%. A perda de eficiência registrada na Fase II foi atribuída ao acúmulo de metabólitos devido à diminuição da concentração de glicose, adicionada como cossubstrato. Porém, são necessários estudos futuros para identificação e remoção biológica de possíveis metabólitos decorrentes da biodegradação dos hidrocarbonetos monoaromáticos.

\section{Referências}

AMERICAN PUBLIC HEALTH ASSOCIATION (APHA). (1995) Standard methods for the examination of water and wastewater. $18 \mathrm{ed}$. Washington: American Public Health Association.

BRASIL. Agência Nacional de Petróleo (ANP). (2009) Anuário estatístico de 2009. Brasília: ANP. Disponível em: <http://www.anp.gov.br/?pg =8240 $\& m=\& t 1=\& t 2=\& t 3=\& t 4=\& a r=\& p s=\&$ cachebust $=127.397 .4171981>$. Acesso em: 23 fev. 2010.

DONAIRE, P.P.R. (2007) Tratamento de água subterrânea contaminada com BTEX utilizando fotocatálise heterogênea. Dissertação (Mestrado em Química) - Universidade Estadual de Campinas, Campinas.

ESPOSITO, E.; AZEVEDO, J.L. (2004) Fungos: uma introdução à biologia, bioquímica e bitecnologia. Caxias do Sul: EDUCS.
FARHADIAN, B.M.; DUCHEZA, D.; VACHELARDA, C.C.; LARROCHEA C. (2008) Monoaromatics removal from polluted water through bioreactors - a review. Water Research, v. 42, p. 1325-1341.

FARIAS, S.L.; PINHEIRO, J.F.; ARAÚJO, R.; RODRIGUES, K.A.; MARINHO, G. (2008) Degradação de benzeno em reatores em batelada com e sem agitação inoculados com Aspergillus niger AN400. III Congresso de Pesquisa e Inovação da Rede Norte Nordeste de Educação Tecnológica. Anais... Fortaleza.

FINOTTI, A.R.; CAICEDO, N.O.L.; RODRIGUES, M.T.R. (2001) Contaminações subterrânea com combustíveis derivados do petróleo: toxidade e a legislação brasileira. Revista Brasileira de Recursos Hídricos, v. 6, n. 2, p. 29-46. 
FORTE, E.J.; AZEVEDO, M.S.; OLIVEIRA, R.C.; ALMEIDA, R. (2007) Contaminação de aqüífero por hidrocarbonetos: estudo e caso na vila Tupi, Porto Velho, Rondônia. Química Nova, v. 30, n. 7, p. 1539-1544.

GRIFFIN, D.H. (1994) Fungal physiology. 2.ed. New York: Wiley-Liss. 458p.

JENNINGS, D.H. (1995) The physiology of fungal nutrition. Cambridge: Cambridge University. 595p.

JERNEC, K.; LEGISA, M. (2004) A drop of intracellular pH stimulates citric acid accumulationby some strains of Aspergillus niger. Journal of Biotechnology, v. 112, p. 289-297.

KENNES, C.; VEIGA, M.C. (2004) Fungal biocatalysts in the biofiltration of VOC-polluted air. Journal of Biotechnology, v. 113, n. 1-3, p. 305-319.

KULKAMP; M.S. (2003) Atenuação natural de hidrocarbonetos de petróleo em um aquífero com derramamento simultâneo de diesel etanol. Dissertação (Mestrado em Engenharia Ambiental) - Departamento de Engenharia Sanitária e Ambiental, Universidade Federal de Santa Catarina, Florianópolis.

KYRIACOU, A.; LASARIDI, K.E.; KOTSOU, M.; BALIS, C.; PILIDIS, G. (2005) Combined bioremediation and advanced oxidation of green table olive processing wastewater. Process Biochemistry, v. 40. p. 1401-1408.

LEMOS, J.L.S.; ARAÚJO, F.M.S. (2002) Isolamento e identificação de fungos degradadores de petróleo.In: X Jornada de Iniciação Científica do CETEM. Anais... Rio de Janeiro.

LOPES, M.; CELESTINO, P.; ANDRADE, M.; MARINHO, G.; RODRIGUES, K. (2008) Remoção biológica de nitrogênio de fósforo de efluente de industria de castanha de caju utilizando reator em batelada. In: II Jornada da Produção Científica e Tecnológica da Região Sul. Anais... Pelotas.

MAZZEO, D.E.C.; LEVY, C.E.; ANGELIS, C.F.; MARIN-MORALES, M.A. (2010) BTEX biodegradation by bacteria from effluents of petroleum refinery. Science of the Total Environmental, v. 418, n. 20, p. 4334-4340.

MALIYEKKAL, S.M.; RENE, E.R.; PHILIP, L.; SWAMINATHAN, T. (2004) Performance of BTX degraders under substrate versatility conditions. $J$ Hazard Mater, v. 109, n. 1-3, p. 201-211.

MELO, I.; GOMES, K.; CUNHA LIMA, P.; RODRIGUES, K.; SAMPAIO,

G. (2009) Tratamento de água residuária sintética por Aspergillus niger
AN400 na remoção de DQO e corante Azo. In: IV Congresso de Pesquisa e Inovação da Rede Norte e Nordeste de Educação Tecnológica CONNEPI Belém.

PAPAGIANNI, M.; MATTEY, M. (2004) Physiological aspects of free and immobilized Aspergillus niger cultures producing citric acid under various glucose concentrations. Process Biochemistry, v. 39, n. 12, p. 1963-1970.

PIETRO, M.B.; HIDALGO, A.; SERRA, J.L.; LLAMA, M.J. (2002) Degradation of phenol by Rhodococcus erithropolis UPV-1 immobilized on biolite in a packed-bed reactor. Journal of Biotechnology, v. 97, p. 1-11.

REINEKE, W. (2001) The Handbook of Environmental Chemistry. v. 2. Biodegradation and Persistence. London: Springer-Verlag Berlin Heidelberg. p. 17.

RODIER, J. (1975) L'analyse de l'eau: eaux naturelles, eaux residuls, eaux de mer. 5. ed. Paris. Dunod, 692 p.

RODRIGUES, K.A. (2006) Uso de reatores biológicos com fungos para remoção de fenol de água residuária sintética. São Carlos. Tese (Doutorado em Hidráulica e Saneamento) - Escola de Engenharia de São Carlos, Universidade de São Paulo, São Carlos.

RODRIGUES, K.A.; SAMPAIO, G.M.M.; ZAIAT, M.; SANTAELA, S.T. (2007) Influência da glicose sobre o consumo de fenol por Aspergillus niger AN 400 em reatores em batelada. Engenharia Sanitária Ambiental, v. 12, n. 2, p. 222-228.

SANTOS, E.O.; ROSA, C.F.C.; PASSOS, C.T.; SANZO, A.V.L.; BURKERT, J.F.M.; KALIL, S.J.; BURKERT, C.A.V. (2008) Pre-screening of filamentous fungi isolated from a contaminated site in Southern Brazil for bioaugmentation purposes. African Journal of Biotechnology, v. 7, n. 9, p. 1314-1317

SILVA, I.E.C.; LUDWIG, C.V.F.; NEUMANN, D.; SCHNEIDER, A,C.; ONOFRE, S.B. (2007) Fungos filamentosos degradadores de compostos fenólicos de água residuária de postos de combustíveis. Revista de Biologia e Saúde da UNISEP, v. 1. n. 1-2, p. 101-108.

SINGH, H. (2006) Mycoremediation: fungal bioremediation. New Jersey: John Wiley \& Sons. 592p.

TIBURTIUS, E.R.L.; PERALTA-ZAMORA, P.; EMMEL, A.; LEAL, E.S. (2005) Degradação de BTXs via processos oxidativos avançados. Química Nova, v. 28, n. 1, p. 61-64. 